\title{
USING SPICE CIRCUIT SIMULATION PROGRAM IN RELIABILITY ANALYSIS OF REDUNDANT SYSTEMS WITH NON-REPAIRABLE UNITS AND COMMON-CAUSE FAILURES
}

\author{
MUHAMMAD TAHER ABUELMA'ATTI ${ }^{\mathrm{a}, *}$ \\ and ISA SALMAN QAMBER ${ }^{\mathrm{b}}$ \\ ${ }^{a}$ King Fahd University of Petroleum and Minerals, Box 203, Dhahran 31261, \\ Saudi Arabia; ${ }^{b}$ University of Bahrain, P.O. Box 32038, Isa Town, Bahrain
}

(Received 10 November 1999; In final form 28 January 2000)

\begin{abstract}
The effectiveness of Simulation Program with Integrated Circuit Emphasis (SPICE) in calculating probabilities, reliability, steady-state availability and mean-time to failure of redundant systems with non-repairable units and common-cause failures described by Markov models is demonstrated. General equations and procedure for constructing the equivalent circuit for $N$ parallel units are presented. Results obtained, for $N=1,2,3$, using SPICE are compared with previously published results obtained using the Laplace transform method. Full SPICE listings are included.
\end{abstract}

Keywords: Reliability; SPICE simulation

\section{INTRODUCTION}

In practice most component failures are hardly statistically independent. Thus, common-cause failure, where a single failure event can propagate and cause failure of more than one unit in the system, has attracted the attention of many investigators in the area of system reliability and availability $[1-8]$. There are several reasons which can lead to common cause failures [5]. Whether it is for predicting the

\footnotetext{
${ }^{*}$ Corresponding author.
} 
behavior of new designs or studying possible changes to existing ones, models of systems with common-cause failures are essential tools for their investigation. Of particular interest here is the Markov analysis technique which is widely used for calculating the reliability and availability of a redundant system with non-repairable units.

Figure 1 shows the state space diagram of a non-repairable $N$ identical unit parallel system with one identical warm standby [2]. As long as one unit is operating normally, the system is considered in an up-state. Figure 1 is obtained on the assumption that common-cause and hardware failures are statistically independent, all failure rates are constant and a common-cause failure can occur from any of the $N+1$ up states of the system. Also, it is assumed that the switching mechanism of the standby is automatic and instantaneous and the standby may fail in its standby mode, in addition to failure in its switching mechanism [1]. The state transition equations associated with the system represented by the state space diagram of Figure 1 can be represented by [1]

$$
\frac{d P_{0}(t)}{d t}=\mu_{2} P_{2}(t)-\left(N \lambda_{1}+\lambda_{2}+\lambda_{c 0}\right) P_{0}(t)
$$

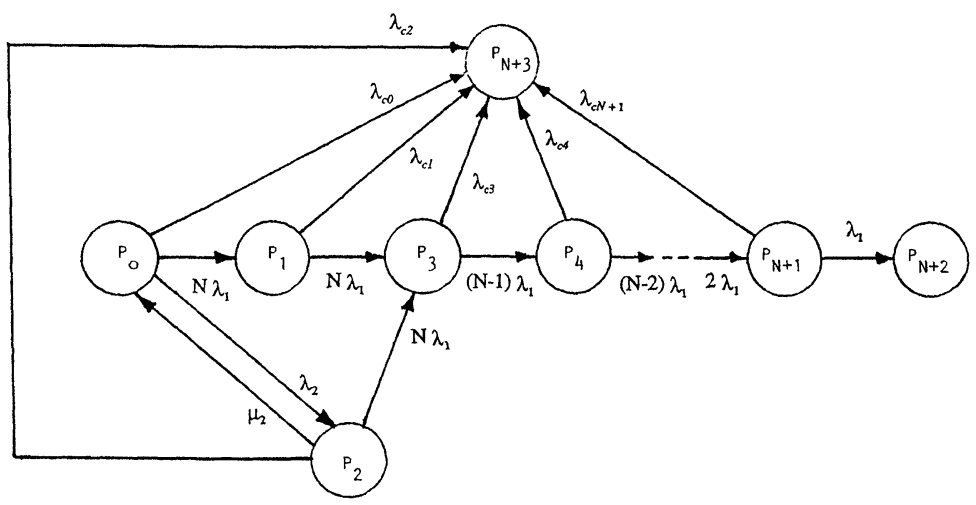

FIGURE 1 State-space diagram of a redundant system comprising $N$ parallel identical units, a warm standby and common-cause failures. $P_{0}: N$ units up and one standby; $P_{1}$ : $N$ units up and one unit fails; $P_{2}: N$ units up and standby fails; $P_{3}: N-1$ units up and two units fail; $P_{4}: N-2$ units up and three units fail; $P_{N+1}$ : One unit up and $N+1$ units fail; $P_{N+2}$ : System fails; $P_{N+3}$ : System fails due to common-cause failure; $\lambda_{1}$ : Constant failure rate of a unit; $\lambda_{2}$ : Constant failure rate of switching mechanism and/or standby itself; $\lambda_{c n}$ : Constant common-cause failure rate from system up-state $n=0,1, \ldots, N+1$; $\lambda_{c 0}$ : Constant critical common-cause failure rate from system state $0 ; \mu_{2}$ : Repairability rate of the warm standby and/or switching mechanism. 


$$
\frac{d P_{1}(t)}{d t}=N \lambda_{1} P_{0}(t)-\left(N \lambda_{1}+\lambda_{c 1}\right) P_{1}(t)
$$

$$
\frac{d P_{2}(t)}{d t}=\lambda_{2} P_{0}(t)-\left(N \lambda_{1}+\mu_{2}+\lambda_{c 2}\right) P_{2}(t)
$$

$$
\frac{d P_{3}(t)}{d t}=N \lambda_{1} P_{1}(t)+N \lambda_{1} P_{2}(t)-\left((N-1) \lambda_{1}+\lambda_{c 3}\right) P_{3}(t)
$$

$$
\frac{d P_{4}(t)}{d t}=(N-1) \lambda_{1} P_{3}(t)-\left((N-2) \lambda_{1}+\lambda_{c 4}\right) P_{4}(t)
$$

$$
\frac{d P_{5}(t)}{d t}=(N-2) \lambda_{1} P_{4}(t)-\left((N-3) \lambda_{1}+\lambda_{c 5}\right) P_{5}(t)
$$

$$
\frac{d P_{N}(t)}{d t}=3 \lambda_{1} P_{N-1}(t)-\left(2 \lambda_{1}+\lambda_{c N}\right) P_{N}(t)
$$

$$
\frac{d P_{N+1}(t)}{d t}=2 \lambda_{2} P_{N}(t)-\left(\lambda_{1}+\lambda_{c N+1}\right) P_{N+1}(t)
$$

$$
\frac{d P_{N+2}(t)}{d t}=\lambda_{1} P_{N+1}(t)
$$

$\frac{d P_{N+3}(t)}{d t}=\lambda_{c 0} P_{0}(t)+\lambda_{c 1} P_{1}(t)+\lambda_{c 2} P_{2}(t)+\cdots+\lambda_{c N+1} P_{N+1}(t)$

where $\left(d P_{n}(t) / d t\right)$ represents the differentiation of the $n$ th-state probability $P_{n}(t)$, at time $t=0, P_{0}(0)=1$ and $P_{k}(0)=0$, for $k=$ $1,2,3, \ldots, N+3$. Equations (1) $-(10)$ can be solved using the Laplace transform method [1-5], yielding expressions for the probability, $P_{n}(t)$, that the overall system under consideration is in state $n$ at time $t$ for $n=0,1, \ldots, N+3$. Alternatively, the steady state probabilities, $P_{n}, n=0,1, \ldots, N+3$ can be obtained by setting the derivatives with respect to time, of Eqs. (1)-(10), equal to zero and using the relationship

$$
\sum_{n=0}^{N+3} P_{n}(t)=1
$$

to solve the resulting $N+4$ algebraic equations. While these approaches are attractive for relatively simple systems, it is virtually impossible to obtain a general time dependent expressions for the 
probabilities of more complicated systems with large values of $N$. Moreover, in some cases recourse to numerical methods may be inevitable in order to solve high order algebraic equations before obtaining the Laplace transforms of the different probabilities.

On the other hand, the Simulation Program with Integrated Circuit Emphasis (SPICE) is a general-purpose program which can be used for d.c., transient and a.c. analysis. Although it was initially developed for integrated circuit analysis, it is now widely used for many nonintegrated circuit applications [9-11]. Recently, the use of SPICE capabilities in studying the behavior of multi-state systems described by Markov models was investigated [12]. The equivalent circuits used and the results obtained for the steady-state probabilities of four-state and five-state models prove that SPICE circuit simulation program is a useful tool for studying the behavior of multi-state systems described by the Markov models.

The major intention of this paper is to present equivalent circuits for studying the behavior of the general non-repairable $N$ identical unit parallel system with one identical warm standby described by Figure 1 and Eqs. (1)-(10). Using the proposed equivalent circuits, reliability and availability analysis of these systems can be easily performed using SPICE. From the SPICE output file the following can be obtained:

(1) The probability, $P_{n}(t)$, that the system is in state $n$ at time $t$.

(2) The system reliability, $R_{s}(t)$, that is the probability that the system is in an up state.

(3) The system steady state availability, $A V_{s s}$, that is the long term probability that the system is in an up state.

(4) The system mean time to failure (MTTF).

\section{PROPOSED MODEL}

According to [12], an appropriate source for the model must be identified and this source-state is recommended to be the equation having the larger number of terms. The source equation is replaced by Eq. (11). It is worth mentioning that, the choice of a particular equation as the source is not critical and any of the state Eqs. (1)-(10) 
can be assigned as the source-state. In the present analysis the source equation is taken as Eq. (1). After replacing Eq. (1) by Eq. (11), performing the necessary modifications and rearrangements, Eqs. (1) $-(10)$, can be rewritten as

$$
\begin{gathered}
P_{0}(t)=1-P_{1}(t)-P_{2}(t)-\cdots-P_{N+3}(t) \\
P_{1}(t)=\alpha_{1}-\alpha_{1} P_{2}(t)-\cdots-\alpha_{1} P_{N+3}(t)-\frac{\alpha_{1}}{N \lambda_{1}} \frac{d P_{1}(t)}{d t} \\
P_{2}(t)=\alpha_{2}-\alpha_{2} P_{1}(t)-\alpha_{2} P_{3}(t)-\cdots-\alpha_{2} P_{N+3}(t)-\frac{\alpha_{2}}{\lambda_{2}} \frac{d P_{2}(t)}{d t} \\
P_{3}(t)=\alpha_{3} P_{1}(t)+\alpha_{3} P_{2}(t)-\frac{\alpha_{3}}{N \lambda_{1}} \frac{d P_{3}(t)}{d t} \\
P_{4}(t)=\alpha_{4} P_{3}(t)-\frac{\alpha_{4}}{(N-1) \lambda_{1}} \frac{d P_{4}(t)}{d t} \\
P_{5}(t)=\alpha_{5} P_{4}(t)-\frac{\alpha_{5}}{(N-2) \lambda_{1}} \frac{d P_{5}(t)}{d t} \\
+\left(\frac{\lambda_{c 2}}{\lambda_{c 0}}-1\right) P_{2}(t)+\left(\frac{\lambda_{c 3}}{\lambda_{c 0}}-1\right) P_{3}(t)+\cdots+\left(\frac{\lambda_{c N+1}}{\lambda_{c 0}}-1\right) \\
+\left(\frac{\lambda_{c N+1}}{\lambda_{c 0}}-1\right) P_{N+1}(t)-P_{N+2}(t)-\frac{d P_{N+3}(t)}{d t} \\
P_{N}(t)=\alpha_{N} P_{N-1}(t)-\frac{\alpha_{N}}{3 \lambda_{1}} \frac{d P_{N}(t)}{d t} \\
P_{N+1}(t)=\alpha_{N+1} P_{N}(t)-\frac{\alpha_{N+1}}{2 \lambda_{1}} \frac{d P_{N+1}(t)}{d t} \\
(t)=1 \\
P_{N+2}(t)=\lambda_{1} \int_{0}^{\infty} P_{N+1}(t) d t
\end{gathered}
$$


where

$$
\begin{aligned}
\alpha_{1} & =\frac{N \lambda_{1}}{2 N \lambda_{1}+\lambda_{c 1}} \\
\alpha_{2} & =\frac{\lambda_{2}}{N \lambda_{1}+\lambda_{c 2}+\lambda_{2}+\mu_{2}} \\
\alpha_{3} & =\frac{N \lambda_{1}}{(N-1) \lambda_{1}+\lambda_{c 3}} \\
\alpha_{4} & =\frac{(N-1) \lambda_{1}}{(N-2) \lambda_{1}+\lambda_{c 4}} \\
\alpha_{5} & =\frac{(N-2) \lambda_{1}}{(N-3) \lambda_{1}+\lambda_{c 5}} \\
\alpha_{N} & =\frac{3 \lambda_{1}}{2 \lambda_{1}+\lambda_{c 4}} \\
\alpha_{N+1} & =\frac{2 \lambda_{1}}{\lambda_{1}+\lambda_{c N+1}} .
\end{aligned}
$$

The electrical equivalent circuit of Eqs. (13)-(21) can be constructed using the subcircuit shown in Figure 2. In the subcircuit, the current is the analog of $P_{n}(t)$, the voltage across the inductor is the

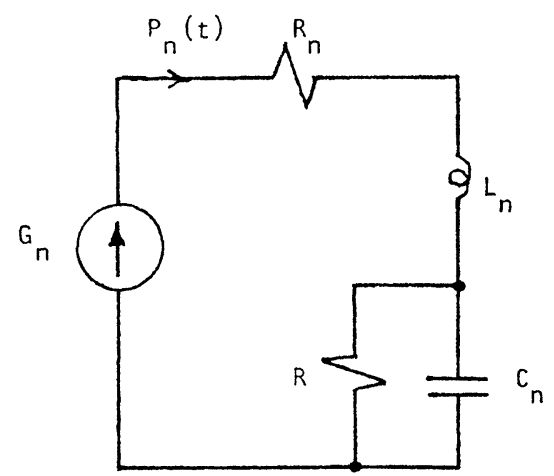

FIGURE 2 Subcircuit used to construct the equivalent circuit of Eqs. (13)-(21) for $n=0,1, \ldots, N+3$. 
analog of the derivative with respect to time, $\left(d P_{n}(t) / d t\right)$, the voltage across the capacitor is the analog of the integration with respect to time, $\int_{0}^{\infty} P_{n}(t) d t$, and the voltage-controlled current source (VCCS), $G_{n}, n=0,1, \ldots, N+3$, represent the right-hand sides of Eqs. (13)(21). The resistor, $R$, in parallel with the capacitor prevent floating node to satisfy PSPICE rules. This resistor is too large to avoid any significant effect on the circuit dynamics. The polynomial feature of SPICE will be used to represent these VCCSs [12]. In the SPICE input file of the circuit of Figure 1, the initial conditions of $P_{n}(t)$, $n=0,1, \ldots, N+3$ are realized by the Initial Condition (IC) option, the transient analysis option (TRAN) will be used to perform the transient analysis of the circuit of Figure 1 and the SPICE output file will contain the probabilities $P_{n}(t), n=0,1, \ldots, N+3$ as a function of time.

Using the probabilities $P_{n}(t), n=0,1, \ldots, N$, the system reliability can be calculated using Eq. (22).

$$
R_{s}(t)=\sum_{n=0}^{M} P_{n}(t)
$$

where $M<N+3$ is the number of states with the system up. The electrical equivalent circuit of Eq. (22) is shown in Figure 3. In the circuit the current is the analog of the system reliability $R_{s}(t)$ and the VCCS, $G_{R}$, represents the right hand-side of Eq. (22).

The steady-state availability, defined as the long term probability

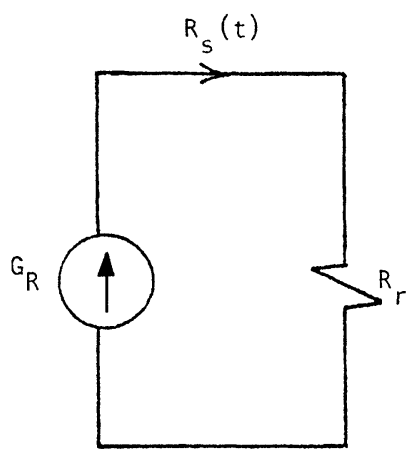

FIGURE 3 Electrical equivalent circuit of Eq. (22) used to calculate the system reliability. 


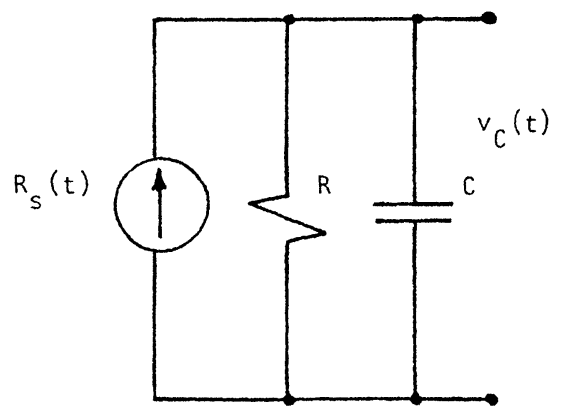

FIGURE 4 Electrical equivalent circuit of Eq. (24) used to calculate the system MTTF.

that the system is in the up state, can be expressed as

$$
A V_{s s}=\sum_{n=0}^{M} P_{n}(\infty)
$$

where $M<N$ is the number of states with the system up. Thus, the steady-state system availability can be obtained directly from the SPICE output file.

The system mean-time to failure can be expressed as

$$
\mathrm{MTTF}=\int_{0}^{\infty} R_{s}(t) d t
$$

The electrical equivalent circuit of Eq. (24) is shown in Figure 4. In the circuit the voltage across the capacitor is the analog of the system MTTF.

\section{EXAMPLES}

The circuits of Figures $2-4$ can be used for obtaining the probabilities $P_{n}(t), n=0,1, \ldots, N+3$, the reliability $R_{s}(t)$, the steady-state availability $A V_{s s}$ and the MTTF of any system described by a set of state transition equations. To illustrate the validity of this approach, two special cases will be considered. 
Case $1 N=1$ and $\mu_{2}=0$. Using Eqs. (1)-(10) and Figure 1, the state transition equations of this case can be written as (Dhillon and Anude, 1993):

$$
\begin{gathered}
\frac{d P_{0}(t)}{d t}=-\left(\lambda_{1}+\lambda_{2}+\lambda_{c 0}\right) P_{0}(t) \\
\frac{d P_{1}(t)}{d t}=\lambda_{1} P_{0}(t)-\left(\lambda_{1}+\lambda_{c 1}\right) P_{1}(t) \\
\frac{d P_{2}(t)}{d t}=\lambda_{2} P_{0}(t)-\left(\lambda_{1}+\lambda_{c 2}\right) P_{2}(t) \\
\frac{d P_{3}(t)}{d t}=\lambda_{1} P_{1}(t)+\lambda_{1} P_{2}(t)
\end{gathered}
$$

and

$$
\frac{d P_{4}(t)}{d t}=\lambda_{c 0} P_{0}(t)+\lambda_{c 1} P_{1}(t)+\lambda_{c 2} P_{2}(t) .
$$

Solving Eqs. (25) - (29) with the aid of Laplace transforms yields the state probabilities. However, this approach is deeply involved in a sea of algebra [2]. Following the procedure described in the previous section, assuming that Eq. (25) is the source equation, Eqs. (25) - (29) can be rewritten as

$$
\begin{gathered}
P_{0}(t)=1-P_{1}(t)-P_{2}(t)-P_{3}(t)-P_{4}(t) \\
P_{1}(t)=\alpha_{1}\left(1-P_{2}(t)-P_{3}(t)-P_{4}(t)\right)-\frac{\alpha_{1}}{\lambda_{1}} \frac{d P_{1}(t)}{d t} \\
P_{2}(t)=\alpha_{2}\left(1-P_{1}(t)-P_{3}(t)-P_{4}(t)\right)-\frac{\alpha_{2}}{\lambda_{2}} \frac{d P_{2}(t)}{d t} \\
P_{3}(t)=\lambda_{1} \int_{0}^{\infty} P_{1}(t) d t+\lambda_{1} \int_{0}^{\infty} P_{2}(t) d t
\end{gathered}
$$

and

$$
P_{4}(t)=\lambda_{c 1} \int_{0}^{\infty} P_{1}(t) d t+\lambda_{c 2} \int_{0}^{\infty} P_{2}(t) d t
$$


or

$$
P_{4}(t)=1+\frac{\lambda_{c 1}-\lambda_{c 0}}{\lambda_{c 0}} P_{1}(t)+\frac{\lambda_{c 2}-\lambda_{c 0}}{\lambda_{c 0}} P_{2}(t)-P_{3}(t)-\frac{1}{\lambda_{c 0}} \frac{d P_{4}(t)}{d t}
$$

where

$$
\alpha_{1}=\frac{\lambda_{1}}{2 \lambda_{1}+\lambda_{c 1}}
$$

and

$$
\alpha_{2}=\frac{\lambda_{2}}{\lambda_{1}+\lambda_{2}+\lambda_{c 2}} .
$$

Equation (34a) is valid for $\lambda_{c 0}=0.0$ and Eq. (34b) is valid for $\lambda_{c 0} \neq 0.0$.

Under the following conditions, $\lambda_{1}=0.0004, \lambda_{2}=0.0001, \lambda_{c 1}=$ $\lambda_{c 2}=0.0003$, then $\alpha_{1}=0.363636, \alpha_{2}=0.125, \quad\left(\alpha_{1} / \lambda_{1}\right)=909.09075$, $\left(\alpha_{2} / \lambda_{2}\right)=1250.0$, and Eqs. (30)-(34) be reduced to

$$
\begin{gathered}
P_{0}(t)=1-P_{1}(t)-P_{2}(t)-P_{3}(t)-P_{4}(t) \\
P_{1}(t)=0.363636\left(1-P_{2}(t)-P_{3}(t)-P_{4}(t)\right)-909.09075 \frac{d P_{1}(t)}{d t} \\
P_{2}(t)=0.125\left(1-P_{1}(t)-P_{3}(t)-P_{4}(t)\right)-1250.0 \frac{d P_{2}(t)}{d t} \\
P_{3}(t)=0.0004 \int_{0}^{\infty} P_{1}(t) d t+0.0004 \int_{0}^{\infty} P_{2}(t) d t
\end{gathered}
$$

and

$$
P_{4}(t)=0.0003 \int_{0}^{\infty} P_{1}(t) d t+0.0003 \int_{0}^{\infty} P_{2}(t) d t
$$

or

$P_{4}(t)=1.0+29.0 P_{1}(t)+29.0 P_{2}(t)-P_{3}(t)-100000.0 \frac{d P_{4}(t)}{d t}$.

Equation (39a) is valid for $\lambda_{c 0}=0$ and Eq. (39b) is valid for $\lambda_{c 0}=0.00001$. 
The equivalent circuit of Eqs. (35)-(39b) is shown in Figure 5 and its SPICE input file is shown in Figure 6 where the initial conditions $P_{n}(0)=0, n=1,2,3,4$ are set to zero. The transient results of Figure 7 show the state probabilities $P_{n}(t)=0, n=0,1, \ldots, 4$ and the system reliability. Table I shows the MTTF for different values of $\lambda_{c 0}$ and $\mu_{2}=0.0006$. Also shown in Table I are the results obtained using the Laplace transform method (Eqs. (45) and (46) of [2]). From Table I

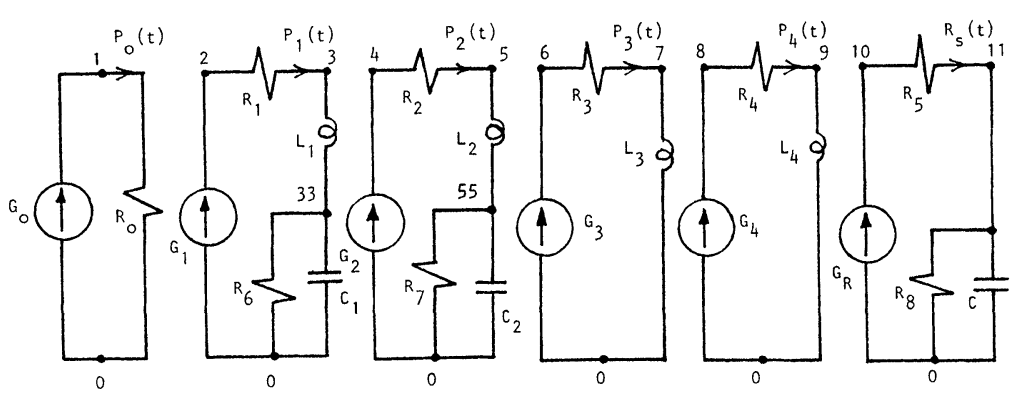

FIGURE 5 Electrical equivalent circuit of Eqs. (25)-(39b) used to calculate probabilities, system reliability and MTTF of Case 1 with $N=1, \mu_{2}=0.0, \lambda_{c 0}=0.00001$.

\begin{tabular}{|c|}
\hline $\begin{array}{llll}\text { R0 } & 1 & 0 & 1 \\
\text { R } & 2 & 3 & 1 \\
\text { R2 } & 4 & 5 & 1 \\
\text { R3 } & 6 & 7 & 1 \\
\text { R } 4 & 8 & 9 & 1 \\
\text { R5 } & 10 & 11\end{array}$ \\
\hline 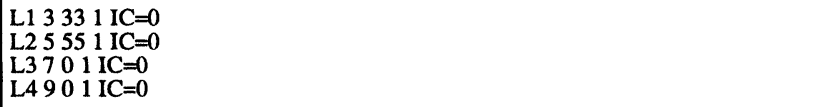 \\
\hline $\begin{array}{lllll}\mathrm{C} 1 & 33 & 0 & 1 \mathrm{IC}=0 \\
\mathrm{R} 6 & 33 & 0 & 1 \mathrm{G} \\
\mathrm{C} 2 & 55 & 0 & 1 \mathrm{IC}=0 \\
\mathrm{R} 7 & 55 & 0 & 1 \mathrm{G} \\
\mathrm{C} 5 & 11 & 0 & 1 \mathrm{IC}=0 \\
\mathrm{R} 8 & 11 & 0 & 1 \mathrm{G}\end{array}$ \\
\hline $\begin{array}{l}\text { G0 } 01 \text { poly(4) } 2344567891-1-1-1-1 \\
\text { G1 } 02 \text { poly(4) } 4567893330.363636-0.363636-0.363636-0.363636-909.09075 \\
\text { G2 } 04 \text { poly(4) } 2367895550.125-0.125-0.125-0.125-1250.0 \\
\text { G3 } 06 \text { poly(2) } 3305500.00 .00040 .0004 \\
\text { G4 } 08 \text { poly(4) } 2345667901.029 .029 .0-1.0-100000.0\end{array}$ \\
\hline Gr 010 poly(3) 1023450.0111 \\
\hline $\begin{array}{l}\text { tran } 1020000 \text { UIC } \\
\text { probe } \\
\text {.plot tran } \mathrm{V}(1,0) \mathrm{V}(2,3) \mathrm{V}(4,5) \mathrm{V}(6,7) \mathrm{V}(8,9) \mathrm{V}(10,11) \mathrm{V}(11,0) \\
\text {.end }\end{array}$ \\
\hline
\end{tabular}

FIGURE 6 SPICE input file for the circuit of Figure 5 corresponding to the state transition diagram of Case 1 with $N=1, \mu_{2}=0.0, \lambda_{c 0}=0.00001$. 


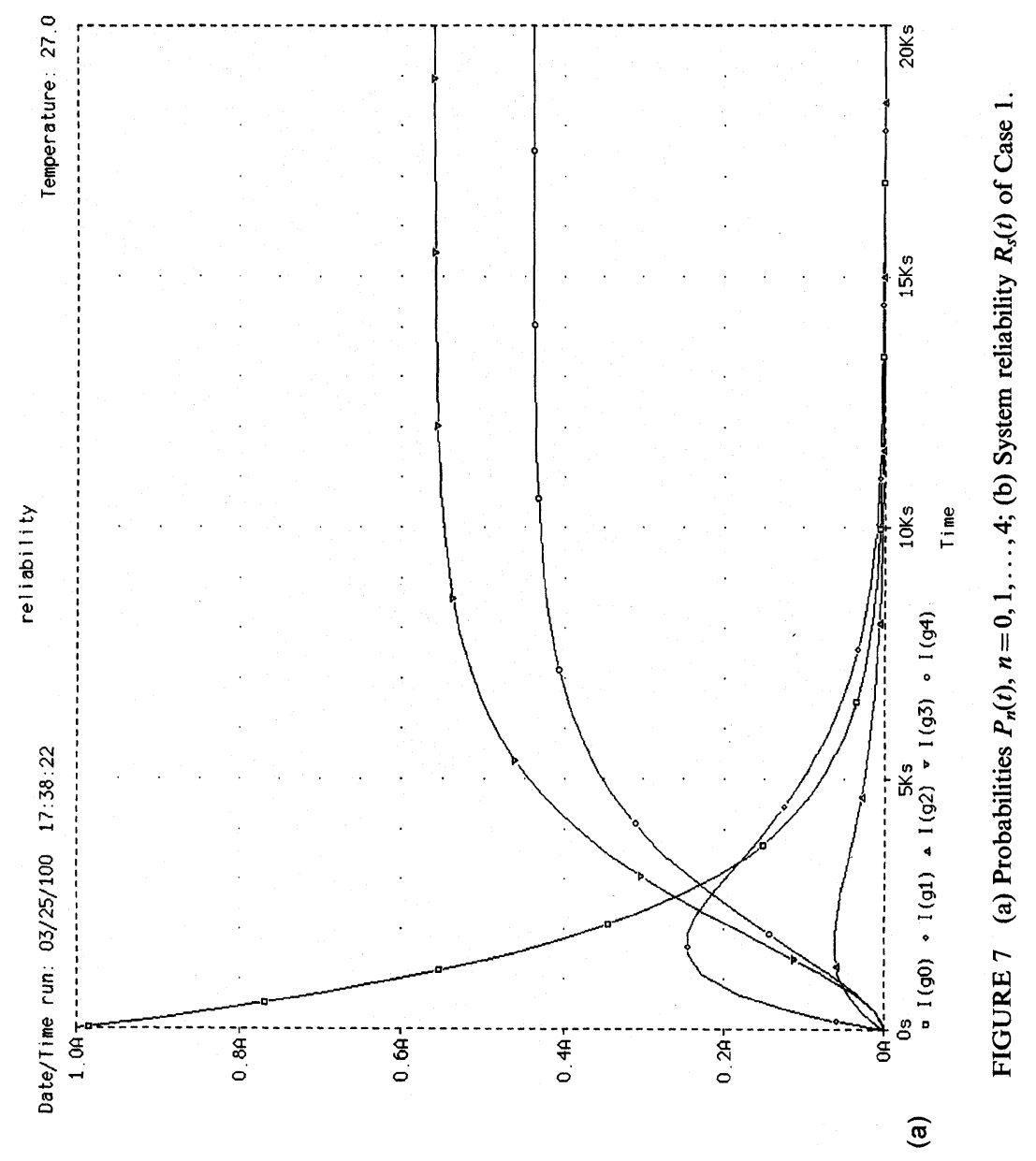




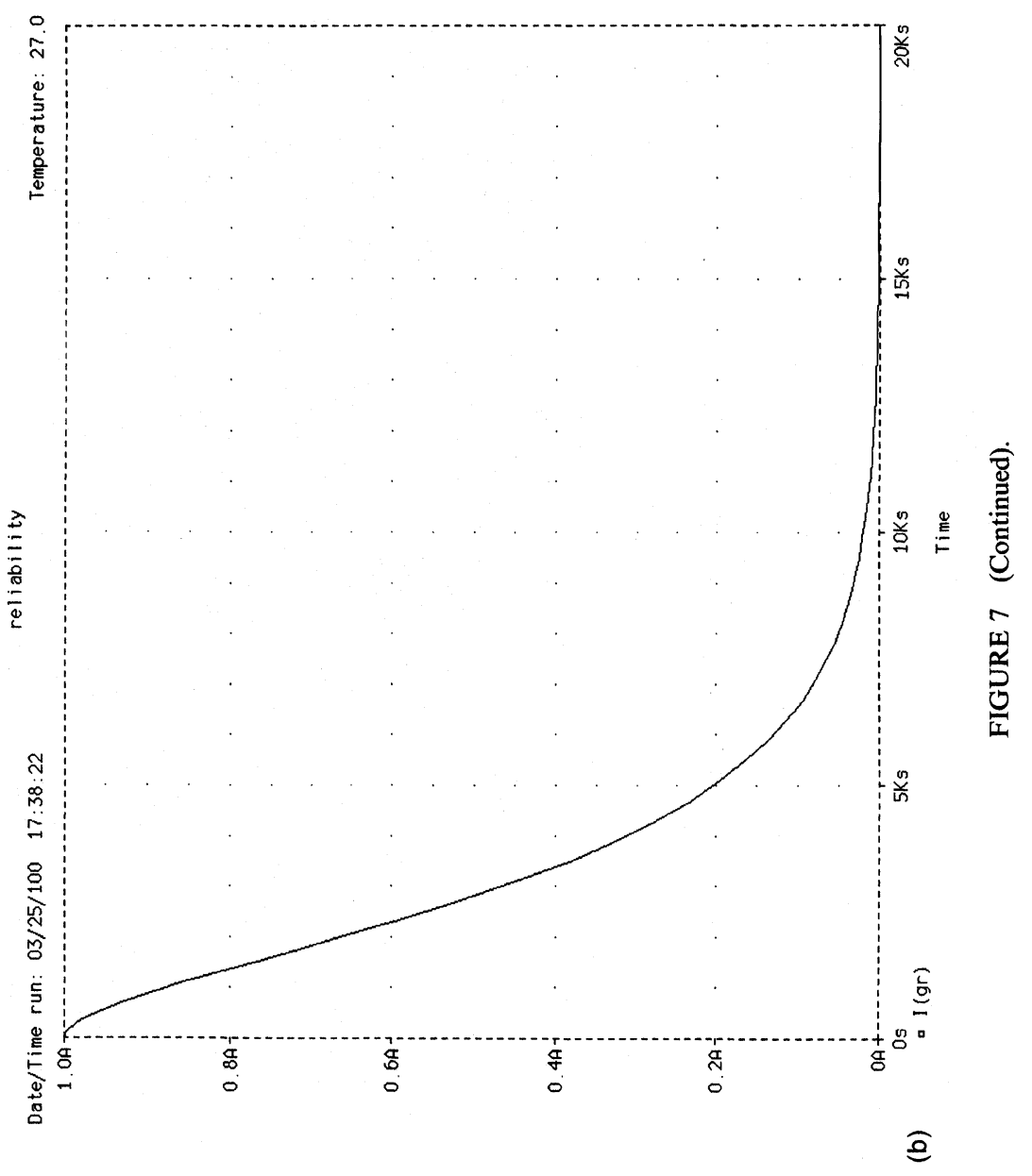


TABLE I MTTF of Case 1 with $N=1, \lambda_{1}=0.0004, \lambda_{2}=0.0001$ and $\lambda_{c 1}=\lambda_{c 2}=0.0003$

\begin{tabular}{|c|c|c|c|c|}
\hline \multirow[b]{2}{*}{$\lambda_{c 0}$} & \multicolumn{2}{|c|}{$\begin{array}{c}M T T F \\
\mu_{2}=0.0\end{array}$} & \multicolumn{2}{|c|}{$\begin{array}{c}M T T F \\
\mu_{2}=0.0006\end{array}$} \\
\hline & $\begin{array}{c}\text { SPICE } \\
\text { simulation }\end{array}$ & $\begin{array}{c}\text { Laplace } \\
\text { transform } \\
\text { method }^{\text {a }}\end{array}$ & $\begin{array}{c}\text { SPICE } \\
\text { simulation }\end{array}$ & $\begin{array}{l}\text { Laplace } \\
\text { transform } \\
\text { method }^{\mathrm{a}}\end{array}$ \\
\hline 0.0000 & 3429 & 3428.6 & 3632 & 3631.5 \\
\hline 0.00001 & 3361 & 3361.3 & 3553 & 3553.2 \\
\hline 0.00002 & 3296 & 3296.7 & 3478 & 3478.3 \\
\hline 0.00003 & 3234 & 3234.5 & 3407 & 3406.4 \\
\hline 0.00004 & 3174 & 3174.6 & 3338 & 3337.4 \\
\hline 0.00005 & 3117 & 3116.9 & 3271 & 3271.2 \\
\hline
\end{tabular}

${ }^{a}$ Dhillon and Anude (1993).

it can be seen that the results obtained using SPICE simulation are in excellent agreement with the results obtained using the Laplace transform method.

Case $2 N=2$ and $\mu_{2}=0$. Using Eqs. (1) $-(10)$ and Figure 1, the state transition equations of this case can be written as [2]:

$$
\begin{gathered}
\frac{d P_{0}(t)}{d t}=-\left(2 \lambda_{1}+\lambda_{2}+\lambda_{c 0}\right) P_{0}(t) \\
\frac{d P_{1}(t)}{d t}=2 \lambda_{1} P_{0}(t)-\left(2 \lambda_{1}+\lambda_{c 1}\right) P_{1}(t) \\
\frac{d P_{2}(t)}{d t}=\lambda_{2} P_{0}(t)-\left(2 \lambda_{1}+\lambda_{c 2}\right) P_{2}(t) \\
\frac{d P_{3}(t)}{d t}=2 \lambda_{1} P_{1}(t)+2 \lambda_{1} P_{2}(t)-\left(\lambda_{1}+\lambda_{c 3}\right) P_{3}(t) \\
\frac{d P_{4}(t)}{d t}=\lambda_{1} P_{3}(t)
\end{gathered}
$$

and

$$
\frac{d P_{5}(t)}{d t}=\lambda_{c 0} P_{0}(t)+\lambda_{c 1} P_{1}(t)+\lambda_{c 2} P_{2}(t)+\lambda_{c 3} P_{3}(t)
$$


Following the procedure described in the previous section, assuming that Eq. (40) is the source equation, Eqs. (40) $-(45)$ can be rewritten as

$$
\begin{gathered}
P_{0}(t)=1-P_{1}(t)-P_{2}(t)-P_{3}(t)-P_{4}(t)-P_{5}(t) \\
P_{1}(t)=\alpha_{1}\left(1-P_{2}(t)-P_{3}(t)-P_{4}(t)-P_{5}(t)\right)-\frac{\alpha_{1}}{2 \lambda_{1}} \frac{d P_{1}(t)}{d t} \\
P_{2}(t)=\alpha_{2}\left(1-P_{1}(t)-P_{3}(t)-P_{4}(t)-P_{5}(t)\right)-\frac{\alpha_{2}}{\lambda_{2}} \frac{d P_{2}(t)}{d t} \\
P_{3}(t)=\alpha_{3}\left(P_{1}(t)+P_{2}(t)\right)-\frac{\alpha_{3}}{2 \lambda_{1}} \frac{d P_{3}(t)}{d t} \\
P_{4}(t)=\lambda_{1} \int_{0}^{\infty} P_{3}(t) d t \\
P_{5}(t)=\lambda_{c 1} \int_{0}^{\infty} P_{1}(t) d t+\lambda_{c 2} \int_{0}^{\infty} P_{2}(t) d t+\lambda_{c 3} \int_{0}^{\infty} P_{3}(t) d t
\end{gathered}
$$

or

$$
\begin{aligned}
P_{5}(t)= & 1+\frac{\lambda_{c 1}-\lambda_{c 0}}{\lambda_{c 0}} P_{1}(t)+\frac{\lambda_{c 2}-\lambda_{c 0}}{\lambda_{c 0}} P_{2}(t) \\
& +\frac{\lambda_{c 3}-\lambda_{c 0}}{\lambda_{c 0}} P_{3}(t)-P_{4}(t)-\frac{1}{\lambda_{c 0}} \frac{d P_{5}(t)}{d t}
\end{aligned}
$$

where

$$
\begin{gathered}
\alpha_{1}=\frac{2 \lambda_{1}}{4 \lambda_{1}+\lambda_{c 1}} \\
\alpha_{2}=\frac{\lambda_{2}}{2 \lambda_{1}+\lambda_{2}+\lambda_{c 2}}
\end{gathered}
$$

and

$$
\alpha_{3}=\frac{2 \lambda_{1}}{\lambda_{1}+\lambda_{c 3}} .
$$

Equation (51a) is valid for $\lambda_{c 0}=0.0$ and Eq. (51b) is valid for $\lambda_{c 0} \neq 0.0$. 
Under the following conditions, $\lambda_{1}=0.0004, \lambda_{2}=0.0001$, $\lambda_{c 1}=\lambda_{c 2}=\lambda_{c 3}=0.0003$, then $\alpha_{1}=0.4210526, \alpha_{2}=0.08333333, \alpha_{3}=$ $1.1428571, \quad\left(\alpha_{1} / 2 \lambda_{1}\right)=526.31575, \quad\left(\alpha_{2} / \lambda_{2}\right)=833.3333, \quad\left(\alpha_{3} / 2 \lambda_{c 3}\right)=$ 1428.5713 and Eqs. $(46)-(51)$ reduce to

$$
\begin{aligned}
& P_{0}(t)=1-P_{1}(t)-P_{2}(t)-P_{3}(t)-P_{4}(t) \\
& P_{1}(t)=0.4210526\left(1-P_{2}(t)-P_{3}(t)-P_{4}(t)-P_{5}(t)\right) \\
& -526.31575 \frac{d P_{1}(t)}{d t} \\
& P_{2}(t)=0.08333333\left(1-P_{1}(t)-P_{3}(t)-P_{4}(t)-P_{5}(t)\right) \\
& -833.3333 \frac{d P_{2}(t)}{d t} \\
& P_{3}(t)=1.1428571\left(P_{1}(t)+P_{2}(t)\right)-1428.5713 \frac{d P_{3}(t)}{d t} \\
& P_{4}(t)=0.0004 \int_{0}^{\infty} P_{3}(t) d t
\end{aligned}
$$

and

$$
P_{5}(t)=0.0003\left(\int_{0}^{\infty} P_{1}(t) d t+\int_{0}^{\infty} P_{2}(t) d t+\int_{0}^{\infty} P_{3}(t) d t\right)
$$

or

$$
\begin{aligned}
P_{4}(t)= & 1.0+29.0 P_{1}(t)+29.0 P_{2}(t) \\
& +29.0 P_{3}(t)-P_{4}(t)-100000.0 \frac{d P_{5}(t)}{d t} .
\end{aligned}
$$

Equation (57a) is valid for $\lambda_{c 0}=0$ and Eq. (57b) is valid for $\lambda_{c 0}=0.00001$.

The equivalent circuit of Eqs. (52)-(57b) is shown in Figure 8 and its SPICE input file is shown in Figure 9 where the initial conditions $P_{n}(0)=0, n=1,2, \ldots, 5$ are set to zero. The transient results of Figure 10 shows the state probabilities $P_{n}(t)=0, n=0,1, \ldots, 5$ and the system reliability. Table II shows the MTTF for different values of $\lambda_{c 0}$ and $\mu_{2}=0.0006$. Also shown in Table II are the results obtained 


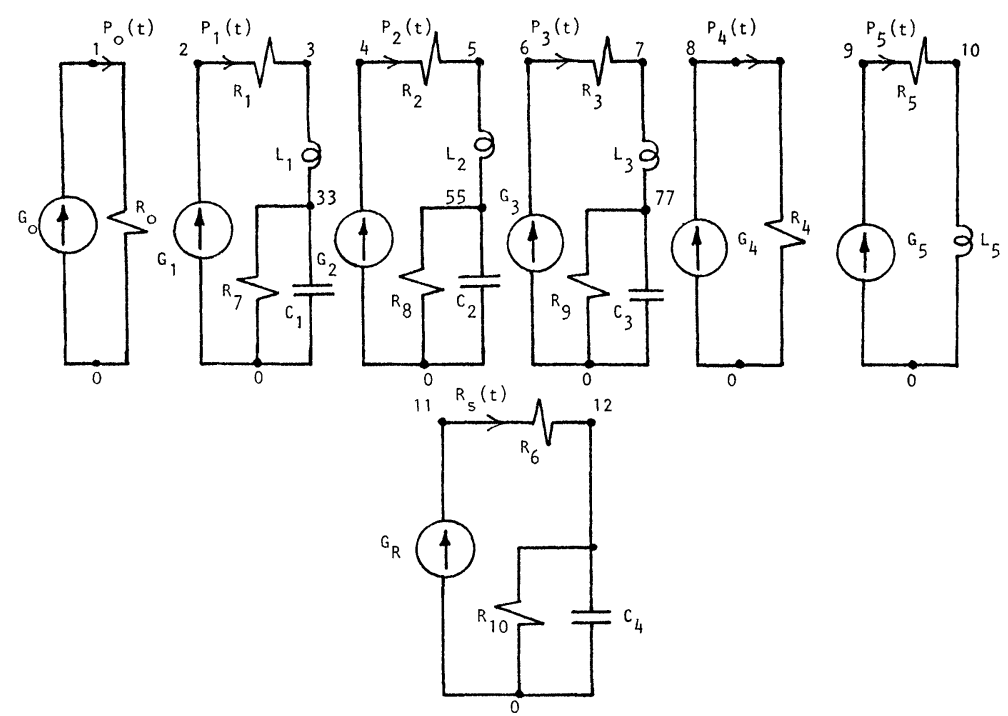

FIGURE 8 Electrical equivalent circuit of Eqs. (25)-(39b) used to calculate probabilities, system reliability and MTTF of Case 2 with $N=2, \mu_{2}=0.0, \lambda_{c 0}=0.00001$.

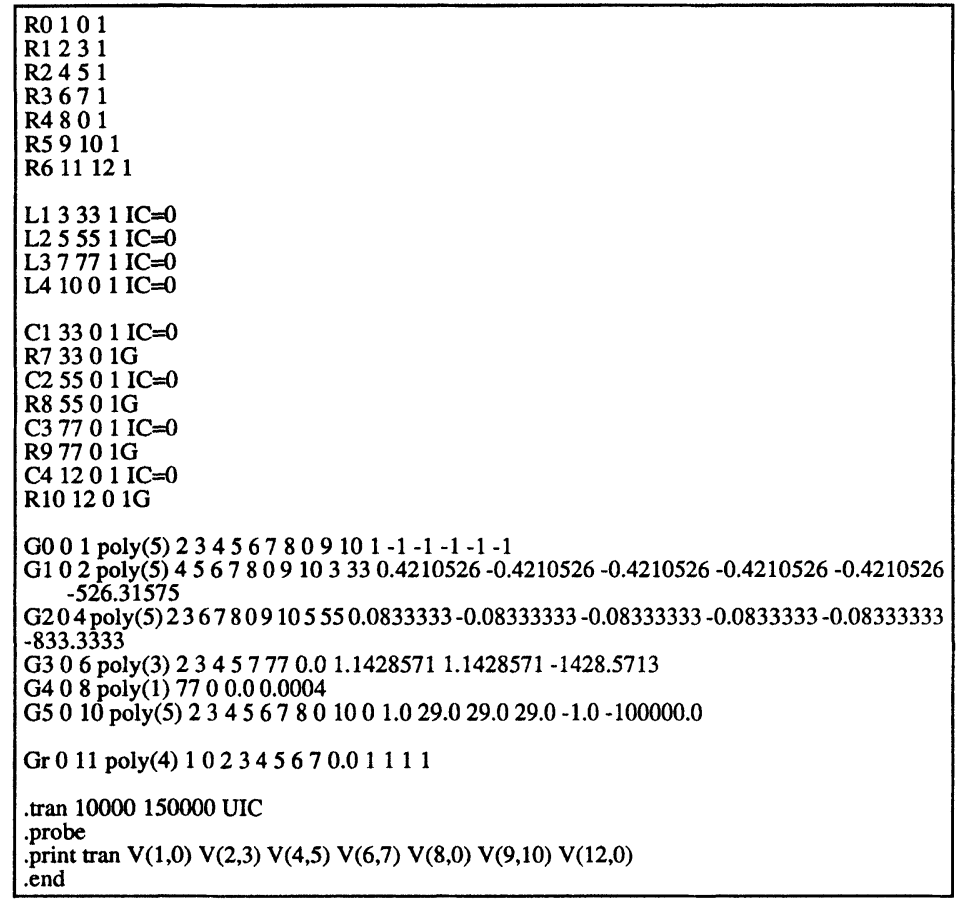

FIGURE 9 SPICE input file of the circuit of Figure 8 corresponding to the state transition diagram of Case 2 with $N=2, \mu_{2}=0.0, \lambda_{c 0}=0.00001$. 


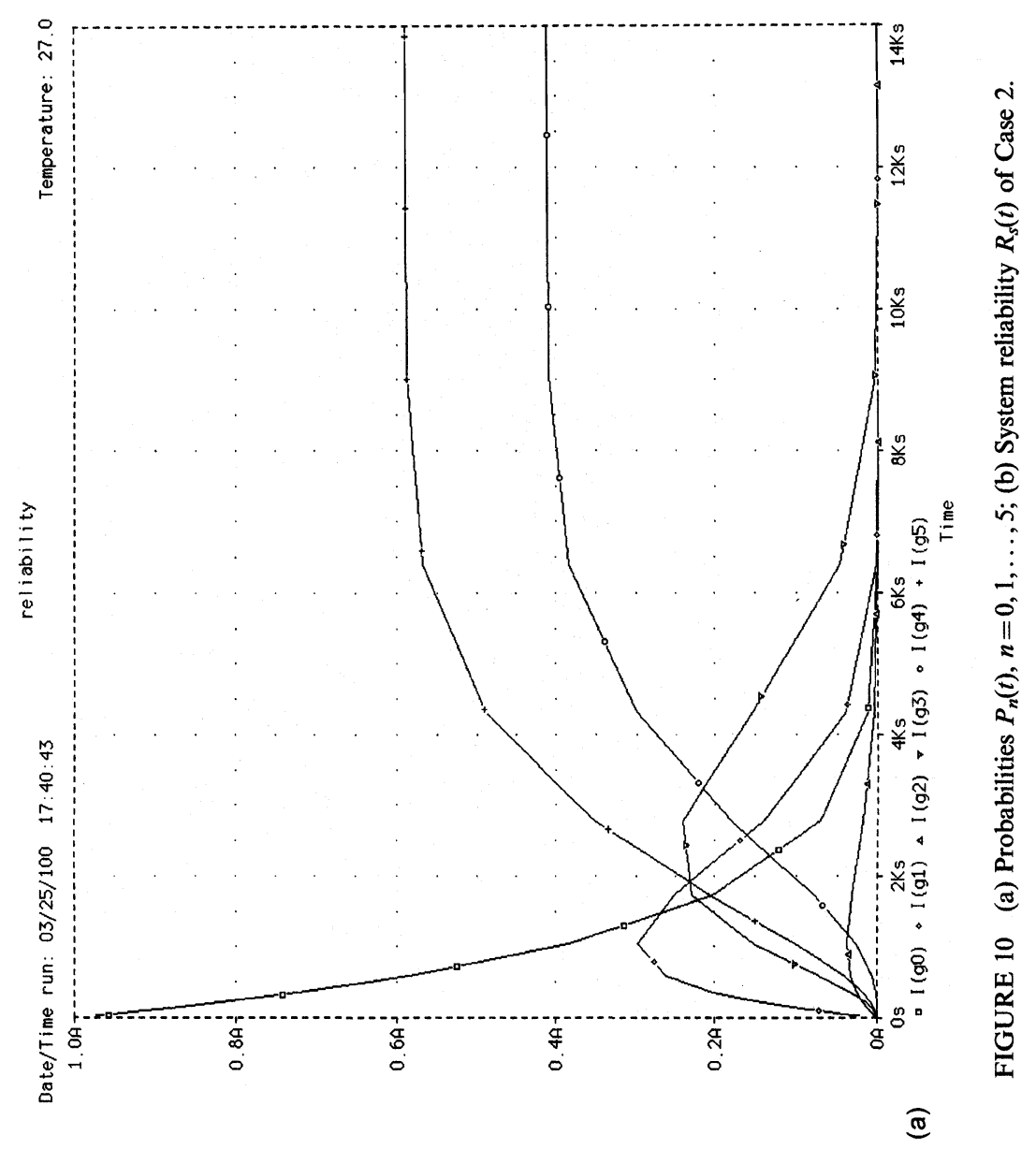




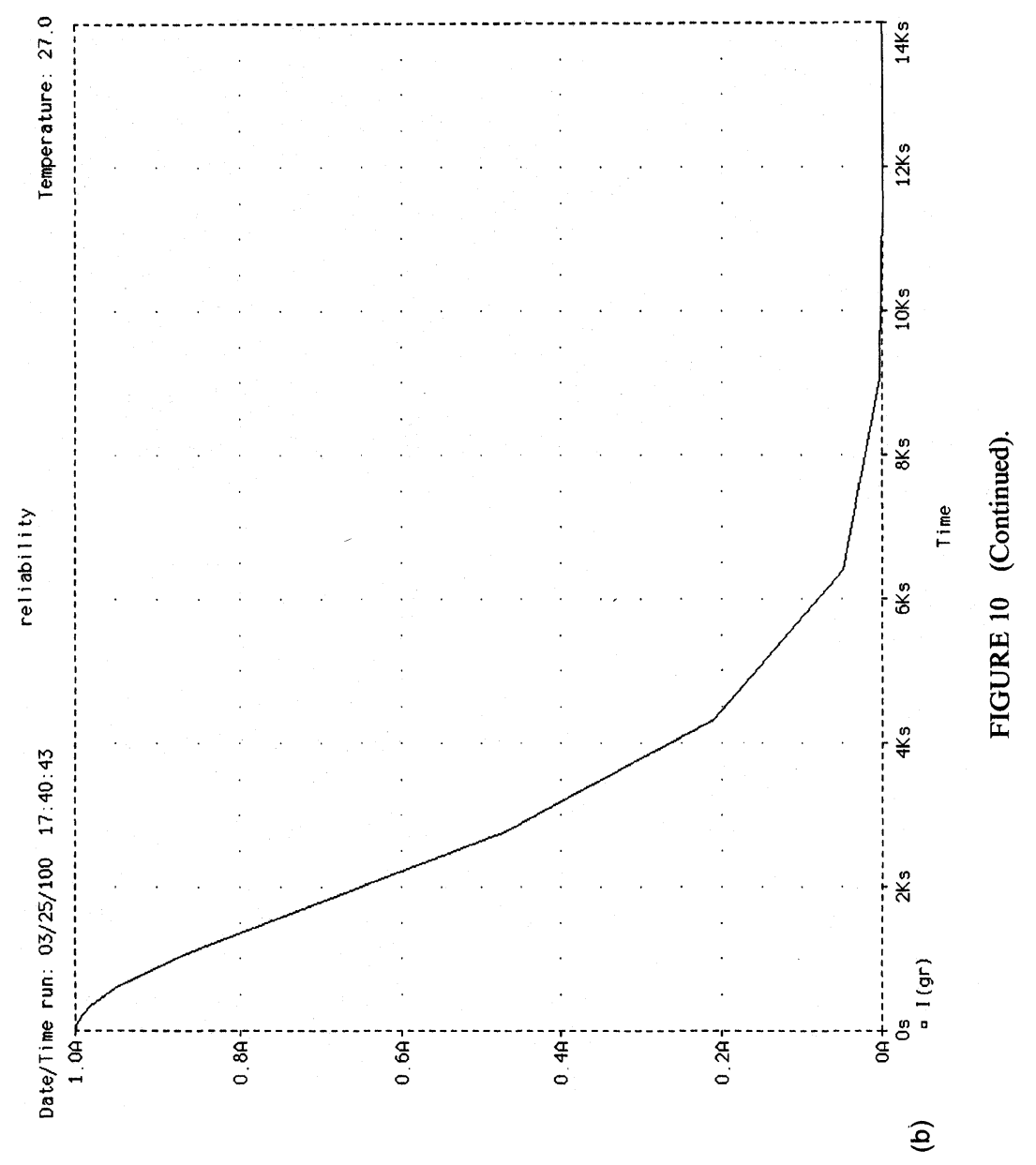


TABLE II MTTF of Case 2 with $N=2, \lambda_{1}=0.0004, \lambda_{2}=0.0001$ and $\lambda_{c 1}=\lambda_{c 2}=$ $\lambda_{c 3}=0.0003$

\begin{tabular}{lccccc}
\hline & \multicolumn{2}{c}{$\begin{array}{c}\text { MTTF } \\
\mu_{2}=0.0\end{array}$} & & \multicolumn{2}{c}{$\begin{array}{c}\text { MTTF } \\
\mu_{2}=0.0006\end{array}$} \\
\cline { 2 - 3 } \cline { 5 - 6 }$\lambda_{c 0}$ & $\begin{array}{c}\text { SPICE } \\
\text { simulation }\end{array}$ & $\begin{array}{c}\text { Laplace } \\
\text { transform } \\
\text { method }^{\text {a }}\end{array}$ & & $\begin{array}{c}\text { SPICE } \\
\text { simulation }\end{array}$ & $\begin{array}{c}\text { Laplace } \\
\text { transform } \\
\text { method }^{\text {a }}\end{array}$ \\
\hline 0.0000 & 3059 & 3059.2 & 3105 & 3104.2 \\
0.00001 & 3025 & 3025.5 & 3069 & 3068.7 \\
0.00002 & 2992 & 2992.7 & 3034 & 3034.0 \\
0.00003 & 2960 & 2960.5 & 3000 & 3000.1 \\
0.00004 & 2929 & 2929.0 & 2967 & 2966.9 \\
0.00005 & 2898 & 2898.0 & 2935 & 2934.5 \\
\hline
\end{tabular}

${ }^{a}$ Dhillon and Anude (1993).

using the Laplace transform method (Eqs. (45) and (46) of [2]). From Table II it can be seen that the results obtained using SPICE simulation are in excellent agreement with the results obtained using the Laplace transform method.

\section{CONCLUSION}

It is evident, from the analysis presented and the examples considered in this paper, that SPICE circuit simulation program is an effective tool for calculating the time dependent probabilities, the system reliability, the system steady-state availability and the mean-time to failure of redundant systems with non-repairable units and commoncause failures. Especially for complicated systems with large number of parallel units, the use of SPICE is highly recommended as it saves time and avoids involvement in a sea of algebra or the possible recourse to numerical solution of high order algebraic equations.

\section{References}

[1] Chung, W. K. (1979). A $N$-unit redundant system with common-cause failures. Microelectronics and Reliability, 19, 377-378.

[2] Chung, W. K. (1989). Reliability analysis of repairable and non-repairable systems with common-cause failure. Microelectronics and Reliability, 29, 545-547.

[3] Dhillon, B. S. (1978). A common-cause failure availability model. Microelectronics and Reliability, 18, 583-584. 
[4] Dhillon, B. S. (1979). A unit redundant system with common-cause failures. IEEE Transactions on Reliability, 19, 267-268.

[5] Dhillon, B. S. (1989). Stochastic analysis of a parallel system with common-cause failures and critical human errors. Microelectronics and Reliability, 29, 627-637.

[6] Dhillon, B. S. and Anude, O. C. (1993). Common-cause failure analysis of redundant system with non-repairable units. Microelectronics and Reliability, 33, $1499-1509$.

[7] Dhillon, B. S. and Viswanath, H. C. (1991). Reliability analysis of a non-identical unit parallel system with common-cause failures. Microelectronics and Reliability, 31, 429-441.

[8] Dhillon, B. S. and Yang, N. (1992). Reliability and availability of warm standby systems with common-cause failures and human errors. Microelectronics and Reliability, 32, 561-575.

[9] Hmurcik, L. V., Hettinger, M., Gottschalck, K. S. and Fitchen, F. C. (1990). SPICE applications to an undergraduate electronic program. IEEE Transactions on Education, 33, 183-189.

[10] Laghari, J. R., Suthar, L. and Cygan, S. (1990). SPICE applications in high voltage engineering education. Computers Education, 14, 455-462.

[11] Prigozy, S. (1989). Novel applications of SPICE in engineering education. IEEE Transactions on Education, 32, 35-38.

[12] Abuelma'atti, M. T. and Qambar, I. S. (1997). SPICE application in the study of the behaviour of multi-state systems described by Markov models. Microelectronics and Reliability, 37, 609-613. 

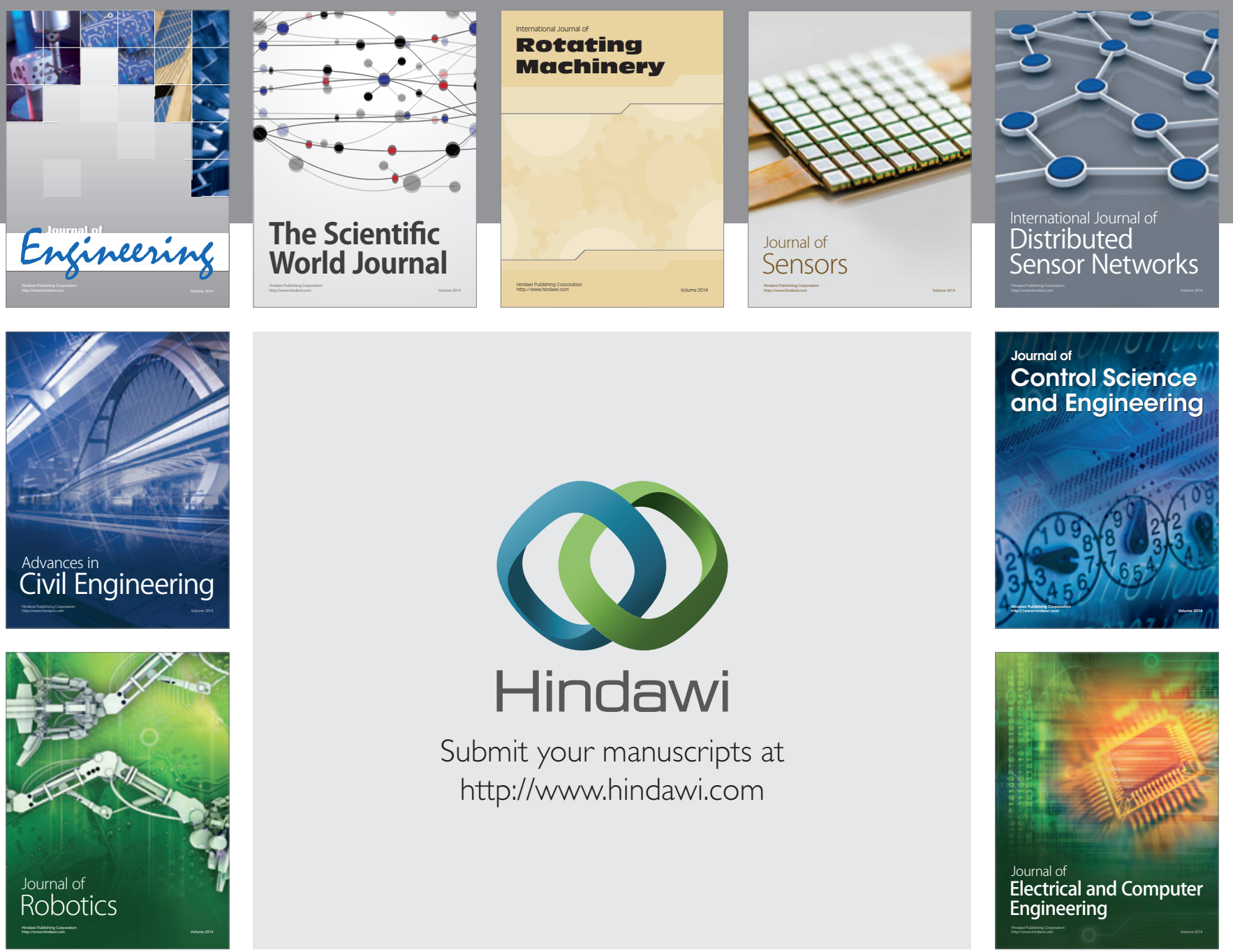

Submit your manuscripts at

http://www.hindawi.com
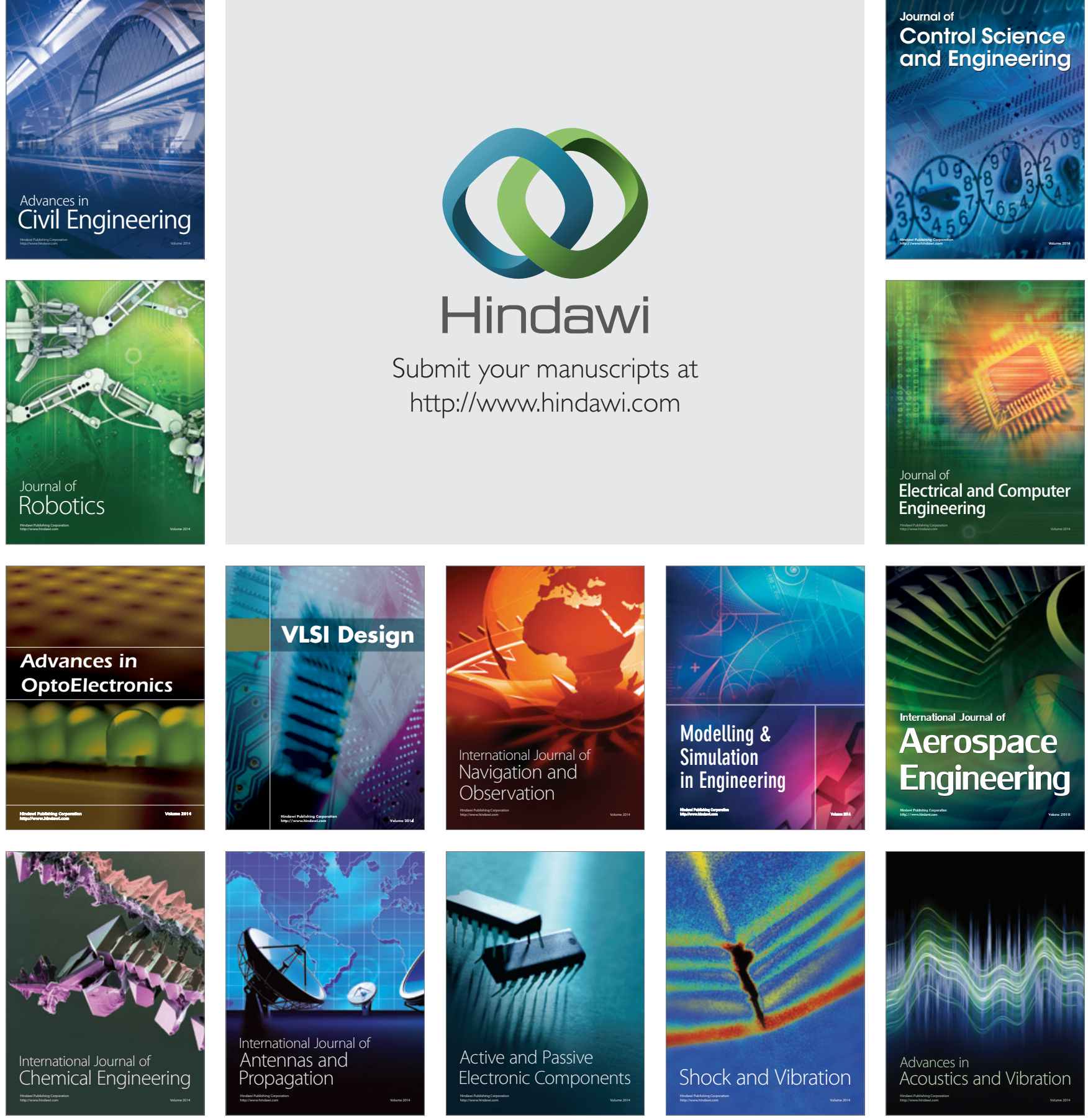